\title{
Study of the twinned dendrite tip shape I: Phase-field modeling
}

\author{
M.A. Salgado-Ordorica*, J.-L. Desbiolles, M. Rappaz \\ Laboratoire de Simulation des Matériaux, Ecole Polytechnique Fédérale de Lausanne, Station 12, 1015 Lausanne, Switzerland
}

\begin{abstract}
The growth kinetics advantage of twinned aluminum dendrites over regular ones is still an unsolved problem of solidification. Although it is linked to the tip geometry, the influence of a coherent (111) twin plane on a $\langle 110\rangle$ twinned dendrite tip is unclear, despite several past experimental observations. In the present contribution, a three-dimensional phase field model implemented on a cluster of parallel computers has been used to simulate the growth of a twinned dendrite under various directional solidification conditions. Only half a dendrite was modeled by replacing the coherent twin plane by a boundary with an appropriate condition on the phase parameter that is equivalent to the Young-Laplace equilibrium condition along the triple line between twinned solid, untwinned solid and liquid. It is found that the small liquid cusp present at the tip rapidly evolves into a doublon-type morphology, i.e. a $\langle 110\rangle$ dendrite split in its center by a deep and thin liquid pool with the triple line at the root. At high growth rates, the two sides of the doublon tend to coalescence and form small isolated liquid droplets. The positive concentration gradient near the doublon root appears to be rapidly smeared out by back-diffusion in the solid, thus making difficult its quantification through experimental methods. These simulation results are correlated with new experimental evidence presented in a companion paper.
\end{abstract}

(C) 2011 Acta Materialia Inc. Published by Elsevier Ltd. All rights reserved.

Keywords: Twinned dendrites; Phase field; Doublon growth; Aluminum alloys

\section{Introduction}

Twinned dendrites owe their name to the fact that, unlike regular dendrites, their trunks are split in their center by a coherent $\left\{\begin{array}{lll}1 & 1 & 1\end{array}\right\}$ plane, while secondary arms meet at an incoherent undulating boundary [2-4]. This creates an altering sequence of twinned and untwinned regions of symmetrically oriented fcc crystal structures that gives to twinned grains (or "feathers") their lamellar appearance. Each pair of lamellae is made of a single dendrite that grows along a $\langle 110\rangle$ direction. Twinned dendrites appear as a defect in semi-continuous casting or directional solidification (DS) of Al alloys. Although their nucleation is still a matter of debate, it is well known that they form in the presence of convection in the melt when solidification is performed under a relatively high thermal gradient $G$ and cooling rate $\dot{T}[5-8]$.

\footnotetext{
* Corresponding author. Present address: Novelis Switzerland SA, Route de Laminoirs 15, CH-3960 Sierre, Switzerland.

E-mail address: mario.salgado@novelis.com (M.A. Salgado-Ordorica).
}

The fact that regular dendrites are often easily overgrown by twinned dendrites during solidification is the consequence of several interconnected factors. Observations of DS specimens with [9] and without quenching [10] indicate that the complex branched morphology of the $\langle 110\rangle$ and $\langle 100\rangle$ arms, their better orientation with respect to the liquidus isotherm and the solutal interactions between them and the side arms of regular dendrites are some of the factors that influence their favorable growth kinetics. Moreover, evidence from previous works $[6,8,10]$ suggests that the tip undercooling of such dendrites might be smaller than that of regular dendrites of the same alloy, due to their shape and the solute rejection associated with it. Beyond these experimental facts, there is another important factor that influences the growth of this particular structures. In order to accommodate the energy of the twin plane, it has been suggested that the shape of the tip of twinned dendrites must be modified with respect to the paraboloid needle shape typically observed for regular dendrites. Based on previous works aimed at measuring the solid-liquid interfacial energy in transparent systems 
$[11,12]$, and treating the two lamellae as two different grains with a strong crystallographic relationship in a low tilt angle boundary, first Chalmers [13], then more specifically Eady and Hogan [14], suggested that the tip would develop a re-entrant angle or a "cusp" in order to satisfy the Smith equation (which is equivalent to the Young-Laplace equilibrium condition at a triple junction) along the triple line (twinned solid/untwinned solid/liquid). This condition is given by:

$2 \gamma_{s \ell} \cos \theta-\gamma_{t}=0$

where $\gamma_{s \ell}$ and $\gamma_{t}$ are the solid-liquid interfacial and twin energies, respectively, and $\theta$ is the dihedral angle formed between the solid-liquid interface and the twin plane (see the top of Fig. 1a [15]). As $\gamma_{t}$ is smaller than $\gamma_{s \ell}$, the twinned dendrite tip would evolve in a stable manner with a small groove, as schematized in Fig. 1a, indicating a compromise between microsegregation and solute pile-up ahead of the interface. The simple two-dimensional (2-D) representation of such a morphology does not take into account the second main curvature radius of the tip, nor the anisotropy of $\gamma_{s \ell}$. To the contrary, many years later, based on numerical simulations, Wood et al. [16] proposed that a sharp (or edgy) dendrite tip would be more advantageous in terms of solute segregation and growth undercooling. They argued that this shape could be equilibrated by torque terms at the triple line (see Fig. 1b). Nevertheless, due to the weak anisotropy of $\gamma_{s \ell}$ experimentally measured in Al alloys [17] and the relatively low thermal gradient predicted by this model for the growth of twinned dendrites, this explanation can probably be ruled out.

More recently, Henry [8] extended Eady and Hogan's hypothesis and suggested the possibility of the existence of a doublon morphology, which is perfectly compatible with the weak anisotropy of $\gamma_{s \ell}$ observed in Al alloys. The experimental evidence found in Al alloys that led to such a conjecture has been discussed in previous works $[8,9]$. Furthermore, such a type of morphology has been observed experimentally in two- and three dimensions on transparent materials $[18,19]$ or predicted theoretically [20-23]. The doublon morphology is schematized in Fig. 1c as a double-tip oriented dendrite that grows with a narrow channel of liquid, a few microns wide at its center. This could also be initiated by the small groove necessary to accommodate the twin energy. However, as solute piles up in the groove, it would make its root recede up to nearly the solidus temperature of the alloy. Under equilibrium conditions, the final solid composition at the root of the liquid channel would be increased to a value close to $C_{0}$ (for $k_{0}<1$ ), instead of being $k_{0} C_{0}$. Thus, such a morphology is believed to induce a strong positive segregation in the doublon trunk center.

\section{The phase field model}

In the present study, the growth of twinned dendrites is simulated using a phase field method [24-26] that was extended to three dimensions from the 2-D PHF-module of the CALCOSOFT ${ }^{\circledR}$ software and implemented on a cluster of distributed memory parallel computers [15]. In order to save computation time and to avoid using a multi-phase field method [27] or an additional orientation parameter [28], only half of the twinned dendrite is modeled with appropriate boundary conditions at the triple line between twinned solid, untwinned solid and liquid.

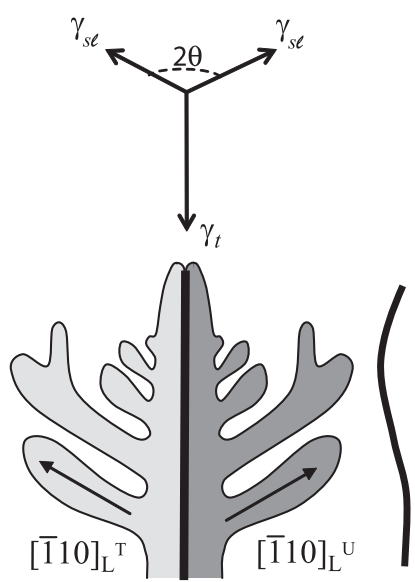

(a)

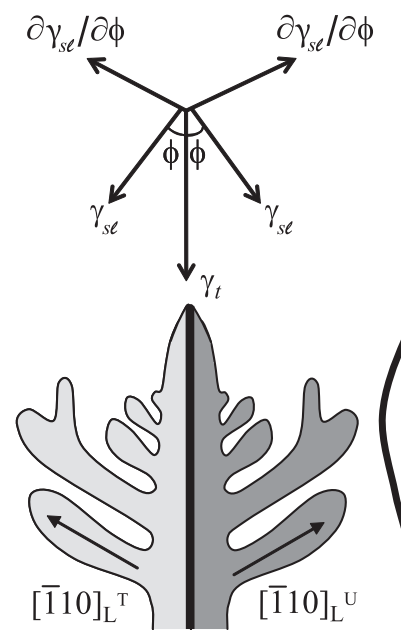

(b)

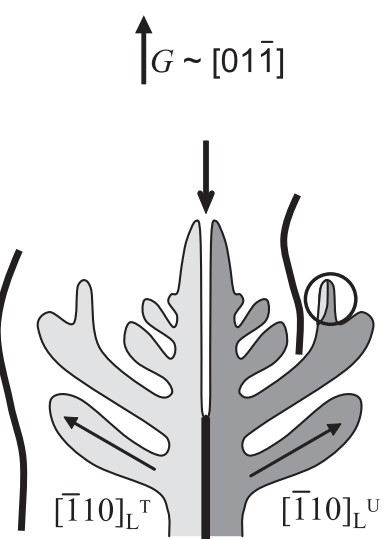

(c)

Fig. 1. Schematic view of twinned dendrite tip morphologies in a plane perpendicular to the twin plane, according to: (a) Eady and Hogan [14]; (b) Wood et al. [16]; and (c) Henry [8]. On the upper parts of ( $a$ and b), the corresponding equilibrium conditions at the triple line have been drawn. The superscripts on the growth directions of $\langle 110\rangle$ side arms indicate that they correspond to the twinned (light gray) and untwinned (dark gray) regions (note that these arms are not exactly in the plane of the figure). The coherent twin plane and undulating boundaries are identified with straight black lines and "S-shape" curves, respectively. The small arrow in (c) indicates the solute pile-up that would create the narrow liquid channel in the center of the twinned dendrite trunk. The lateral twin propagation mechanism is encircled at the right tertiary arm of the doublon morphology in (c) [10]. 
From a thermodynamic point of view, as described by Boettinger et al. [26], the free energy functional $F$ of the considered domain $V$ is given by:

$F=\int_{V}\left[f(T, C, \phi)+\frac{\varepsilon^{2}}{2}|\nabla \phi|^{2}\right] d V$

where $f$ is the volumetric local free energy, constructed from the free energies of the individual elements and their solutions in both the solid and liquid phases. Thus, it is a function of temperature $T$, solute composition $C$ and phase field $\phi$. In the present work, the scalar variable $\phi$ evolves continuously from 1 in the solid to 0 in the liquid across an interface of thickness $\delta$. The parameter $\varepsilon$ appearing in Eq. 2 is related to the solid-liquid interfacial energy $\gamma_{s \ell}$ and $\delta$. The anisotropy of $\gamma_{s \ell}$ has been introduced here through the parameter $\varepsilon$ :

$\varepsilon(\mathbf{n})=\bar{\varepsilon} \eta(\mathbf{n})$

where $\mathbf{n}=\left(n_{x}, n_{y}, n_{z}\right)$ is the unit vector normal to the solidliquid interface in the crystallographic reference frame and $\eta$ is a function that describes the orientation dependence of $\gamma_{s \ell}$. For materials with a cubic structure, the equivalent form of the spherical harmonics that respect the cubic symmetry of the fcc structure is expressed as $[29,30]$ :

$$
\begin{aligned}
\eta(\mathbf{n})= & 1+a_{1}(Q-3 / 5)+a_{2}(3 Q+66 S-17 / 7) \\
& +a_{3}\left(65 Q^{2}-94 Q-208 S+33\right)+\cdots
\end{aligned}
$$

with $Q=n_{x}^{4}+n_{y}^{4}+n_{z}^{4}$ and $S=n_{x}^{2} n_{y}^{2} n_{z}^{2}$. While $a_{1}>0$ and $a_{2}=a_{3}=0$ produce $\langle 100\rangle$ dendrites, $a_{1}=a_{3}=0$ and $a_{2}<0$ induces a dendrite growth along the $\langle 110\rangle$ directions $[31,32]$. In previous works, it has been shown that the parameter $a_{1}$ is weak [17] in $\mathrm{Al}-\mathrm{Cu}$ alloys, and also that a continuous dendrite orientation transition from $\langle 100\rangle$ to $\langle 110\rangle$ can be induced when highly anisotropic solute elements such as zinc are added [33]. In the particular case of twinned dendrites whose growth has been clearly determined to be along $\langle 110\rangle$ directions [3,9], we have simply set $a_{1}=a_{3}=0$ and $a_{2}<0$.

The phase-field evolution equation when the interfacial energy is anisotropic is given by [26]:

$$
\begin{aligned}
\frac{\partial \phi}{\partial t} & =-M_{\phi} \frac{\delta F}{\delta \phi} \\
& =-M_{\phi}\left[\frac{\partial f}{\partial \phi}-\nabla \cdot\left(\varepsilon^{2} \nabla \phi\right)+\nabla \cdot|\nabla \phi|^{2} \varepsilon \frac{\partial \varepsilon}{\partial(\nabla \phi)}\right]
\end{aligned}
$$

where $M_{\phi}$ is the mobility coefficient related to the interface kinetic coefficient and $\frac{\delta F}{\delta \phi}$ is the functional derivative of $F$.

The solute diffusion equation in its simplest form was derived by Tiaden et al. [27] for a multi-phase field model. For a solid-liquid transformation, and assuming that the partition coefficient $k_{0}$ is constant, the solute diffusion equation can be written as:

$$
\frac{\partial C}{\partial t}=\nabla \cdot\left[\bar{D}\left(\nabla C+\frac{\left(1-k_{0}\right) C}{1-\phi+k_{0} \phi} \nabla \phi\right)\right]
$$

with

$\bar{D}=D_{s}+\frac{1-\phi}{\phi k_{0}+(1-\phi)}\left(D_{\ell}-D_{s}\right)$ and $C=C_{\ell}(1-\phi)+k_{0} C_{\ell} \phi$

where $D_{s}$ and $D_{\ell}$ are the diffusion coefficients in the solid and the liquid, respectively, $\bar{D}$ is an average diffusion coefficient and $C_{\ell}$ is the liquid composition.

Within the phase field approach described so far, the enhanced non-equilibrium effects associated with a thick solid-liquid interface are still not corrected. Such effects translate into the artificial trapping of atoms that cannot escape the advancing solidification front fast enough to maintain equilibrium at the interface. An anti-trapping current term, $\mathbf{j}$, introduced by Karma [34], was therefore included in the model. It can be written as:

$\mathbf{j}=\delta \frac{\left(1-k_{0}\right) C}{1-\phi+k_{0} \phi} \frac{\partial \phi}{\partial t} \frac{\nabla \phi}{\|\nabla \phi\|}$

The overall term $\mathbf{j}$ is finally added in the divergence operator on the right-hand side of Eq. 6. This model has been successfully used for the simulation of dendritic growth in hot-dipped galvanized coatings [35,36], for the growth of regular dendrites in the Al-Zn system [31] and of the preliminary stages of the growth of twinned dendrites [15] (in all cases without the anti-trapping current term).

\section{Boundary conditions and solidification parameters}

The calculation domain and the boundary condition implemented at one of its boundaries to simulate the coherent twin plane are shown in Fig. 2. The calculation domain is reduced to a quarter of a twinned dendrite, with the $(11 \overline{1})$ twin plane corresponding to the right $y z$-boundary and the thermal gradient $G$ lying within any $x y$-plane at an angle $\alpha$ with respect to the $y$-axis. The boundary condition set on this $y z$-plane for the phase parameter was an extension to three dimensions of the method used by Sémoroz et al. [35] for the simulation of 2-D dendrite growth in thin coatings. Instead of the condition $\nabla \phi \cdot \mathbf{n}_{\mathbf{b}}=0$ (where $\mathbf{n}_{\mathbf{b}}$ is the normal to the boundary) usually set for a symmetry plane, a condition $\nabla \phi \cdot \mathbf{n}_{\mathbf{b}}=\|\nabla \phi\| \cos \theta^{\prime}$ was imposed (note the change of sign due to the convention used for $\phi$ in the solid and the liquid). Such a condition implicitly assumes that the solid-liquid interfacial energy $\gamma_{s \ell}(\mathbf{n})$ is isotropic, which is a reasonable assumption for the very weak anisotropy exhibited by aluminum alloys [17]. ${ }^{1}$ The angle $\theta^{\prime}$ remained constant throughout the calculation and was measured in the solid; for example, an angle larger than $90^{\circ}$ corresponds to a grooved dendrite tip. This "wetting" angle of the solid on the $y z$-plane is equal to the complement of half the dihedral angle measured at the triple line between the twinned solid, untwinned solid and liquid.

\footnotetext{
${ }^{1}$ When $\gamma_{s \ell}$ is very anisotropic, its orientation dependence must be accounted for [37].
} 


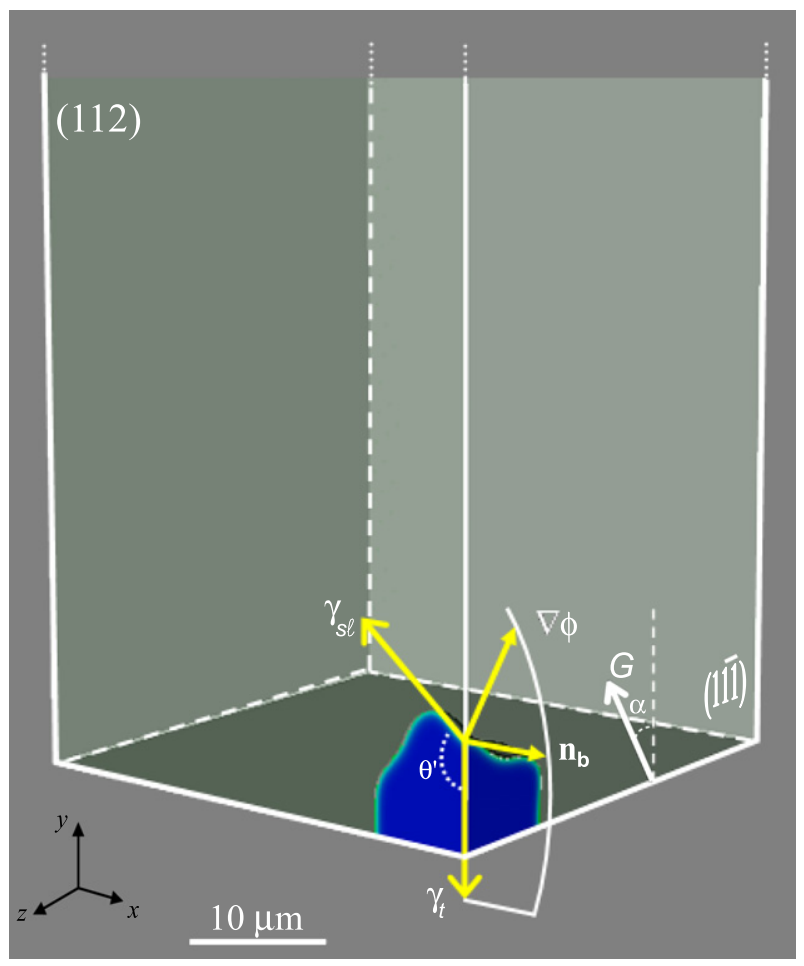

Fig. 2. Calculation domain with the imposed thermal gradient and the Young-Laplace equation imposed at the right $y z$ boundary, identified as the $\left(\begin{array}{lll}1 & 1 & \overline{1}\end{array}\right)$ coherent twin plane. Only a quarter of a hemispherical nucleus, corresponding to half of the twinned part of a twinned dendrite, is simulated. The (112) plane is perpendicular to the (11 $\overline{1})$ plane.

At the beginning of the calculation, a quarter of a nucleus, corresponding only to the twinned region of such a dendrite, was located at the bottom-right corner of the domain. Its initial undercooling $\Delta T$ was set to $5 \mathrm{~K}$ and its anisotropy parameters were $a_{1}=a_{3}=0, a_{2}=-0.0172$ (Eq. 4). The constant thermal gradient $G \approx 1 \times 10^{5} \mathrm{~K} \mathrm{~m}^{-1}$ was one order of magnitude larger than the experimental value [1] in order to compress the mushy zone and to obtain realistic simulation times. Although it is not necessary to model the entire mushy zone, it will be shown that the grooved geometry of the twinned dendrite tip makes the triple line recede due to solute pile-up. Increasing the thermal gradient reduces the distance between the dendrite tip and the triple line position, and thus the size of the computation domain along the $z$-axis. A constant cooling rate $\dot{T}$ was also imposed, thus allowing the temperature of each point of the domain to be updated at each time step.

Typical properties of the alloy used for these simulations (namely $\mathrm{Al}-9$ at.\%Zn) are shown in Table 1, whereas Table 2 summarizes the solidification conditions and the simulation parameters relative to each figure that will be discussed. Note that, in Table 1, $\Gamma$ is the Gibbs-Thomson coefficient, $L_{f}$ is the volumetric latent heat of fusion, $k_{0}$ is the partition coefficient, $T_{f}$ is the melting point of pure aluminum and $m_{\ell}$ is the slope of the liquidus line. In Table 2, $C_{0}$ is the initial composition of the alloy, $G$ is the thermal gradient, $\dot{T}$ is the cooling rate, $v_{T}=|\dot{T}| / G$ is the isotherm velocity, $t$ is the simulated time and $t_{\mathrm{CPU}}$ is the CPU time. The number of processors (\# Proc.) used for each calculation is also specified in Table 2. In all cases, the cell size $\Delta x=0.1 \mu \mathrm{m}$, and the time step $\Delta t=5 \times 10^{-7} \mathrm{~s}$.

\section{Results and discussion}

\subsection{Quantitative assessment of the model}

To make a quantitative assessment of the morphologies obtained with the present model, a comparison of 3-D simulations was performed with and without the solute antitrapping current term $\mathbf{j}$ in Eq. 6. The conditions selected for this computation are indicated in Table 2. For this preliminary study, the complement of the dihedral angle $\theta^{\prime}$ was set to $90^{\circ}$, i.e. growth of a regular $\langle 110\rangle$ dendrite is expected.

The 3-D views ${ }^{2}$ of the composition distribution profiles after $0.11 \mathrm{~s}$ of simulation are shown in Fig. $3 \mathrm{a}$ and $\mathrm{b}$ for $\mathbf{j}=0$, and $\mathbf{j} \neq 0$, respectively. A comparison of the position of the $\langle 110\rangle$ dendrite tip indicates that the undercooling is slightly lower when the anti-trapping term is neglected $\left(\Delta T_{\text {tip }}=5.26\right.$ and $5.66 \mathrm{~K}$ for $\mathbf{j}=0$, and $\mathbf{j} \neq 0$, respectively). By taking the minimum and maximum values of composition $C$ along the solid-liquid interface at the dendrite tip position, an effective partition coefficient $k_{0}^{*}$ of 0.404 is obtained when $\mathbf{j}=0$. This is about $6.6 \%$ larger than the value introduced into the model (see Table 1). When the anti-trapping term is introduced, $k_{0}^{*}=0.383$, only $2.1 \%$ above the imposed value of $k_{0}$. The corresponding instantaneous dendrite tip velocities, calculated from the length covered by the dendrite during $\Delta t=0.01 \mathrm{~s}$, are $0.52 \mathrm{~mm} \mathrm{~s}^{-1}$ and $0.47 \mathrm{~mm} \mathrm{~s}^{-1}$, respectively, which are very close to the imposed solidification velocity $v_{s} \approx v_{T}=$ $0.5 \mathrm{~mm} \mathrm{~s}^{-1}$.

\subsection{Effect of the dihedral angle}

To evaluate the effect of the imposed boundary condition on the dendrite morphology, 2-D simulations ${ }^{3}$ were performed in the $x y$ plane of Fig. 2 (also identified as the (112) plane). In this case, the direction of $G$ was aligned with the vertical axis $(\alpha=0)$. Fig. 4 shows the isovalue $\phi$ $=0.5$ after $0.11 \mathrm{~s}$ for different values of $\theta^{\prime}$ imposed on the vertical, right twin boundary, as indicated in the figure. Note that these instantaneous growth morphologies do not correspond yet to a steady state. As solidification proceeds, the dendrite tip tends to grow away (split) from the twin plane when $\theta^{\prime}>90^{\circ}$. A small groove first forms due to the imposed equilibrium condition at the boundary. This

\footnotetext{
${ }^{2}$ All the views of the composition and the phase fields reported in this work were obtained with the open-source visualization software Paraview (www.paraview.org).

${ }^{3}$ Note that calculations were actually performed in a 3-D domain reduced to a layer of only two cells along the $z$ axis. The nucleus at the onset of the calculation has a cylindrical, instead of a spherical geometry.
} 
Table 1

Thermophysical properties of an Al-9 at. $\% \mathrm{Zn}$.

\begin{tabular}{|c|c|c|c|c|c|c|}
\hline$D_{s}\left(\mathrm{~m}^{2} \mathrm{~s}^{-1}\right)$ & $D_{\ell}\left(\mathrm{m}^{2} \mathrm{~s}^{-1}\right)$ & $\Gamma(\mathrm{K} \mathrm{m})$ & $L_{f}\left(\mathrm{~J} \mathrm{~m}^{-3}\right)$ & $k_{0}$ & $T_{f}\left({ }^{\circ} \mathrm{C}\right)$ & $m_{\ell}($ K/at. $\%)$ \\
\hline $1.16 \times 10^{-13}$ & $2.7 \times 10^{-9}$ & $2.3 \times 10^{-7}$ & $1 \times 10^{9}$ & 0.375 & 660 & -3.57 \\
\hline
\end{tabular}

Table 2

Solidification conditions and simulation parameters.

\begin{tabular}{|c|c|c|c|c|c|c|c|c|c|}
\hline Fig. (-) & Domain $\left(\mu \mathrm{m}^{3}\right)$ & $C_{0}($ at. $\%)$ & $k_{0}(-)$ & $G\left(\mathrm{~K} \mathrm{~m}^{-1}\right)$ & $\dot{T}\left(\mathrm{~K} \mathrm{~s}^{-1}\right)$ & $v_{T}\left(\mathrm{~mm} \mathrm{~s}^{-1}\right)$ & $t(\mathrm{~s})$ & $t_{\mathrm{CPU}}(\mathrm{h})$ & \# Proc. (-) \\
\hline Fig. 3 & $30 \times 60 \times 30$ & 9 & 0.375 & $1 \times 10^{5}$ & -50 & 0.5 & 0.11 & 52 & 64 \\
\hline Fig. 4 & $10 \times 40 \times 2$ & 9 & 0.375 & $1 \times 10^{5}$ & -70 & 0.7 & 0.11 & 1.3 & 16 \\
\hline Fig. 5 & $30 \times 100 \times 30$ & 9 & 0.375 & $1 \times 10^{5}$ & -70 & 0.7 & 0.104 & 92 & 64 \\
\hline Fig. 8 & $30 \times 140 \times 34$ & 9 & 0.375 & $1 \times 10^{5}$ & -70 & 0.7 & 0.152 & 192 & 64 \\
\hline Fig. 8 & $30 \times 140 \times 34$ & 4 & 0.5 & $1 \times 10^{5}$ & -70 & 0.7 & 0.152 & 192 & 64 \\
\hline Fig. 9 & $30 \times 100 \times 34$ & 4 & 0.5 & $1 \times 10^{5}$ & -70 & 0.7 & 0.08 & 71 & 64 \\
\hline
\end{tabular}

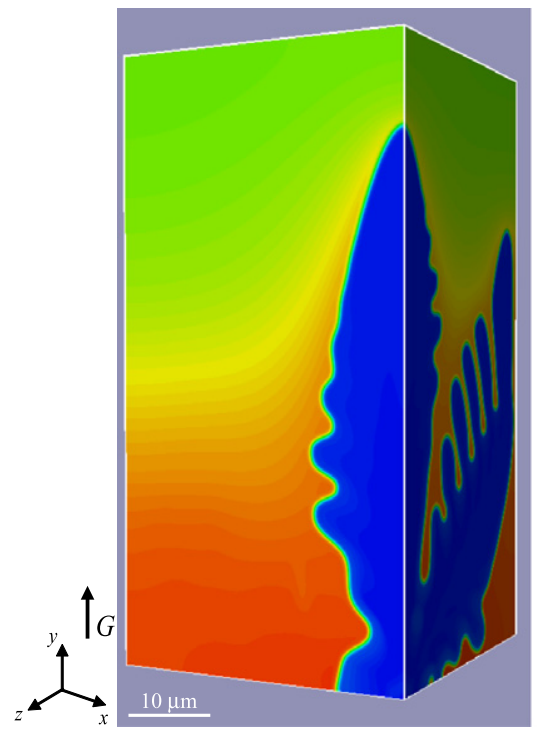

(a)

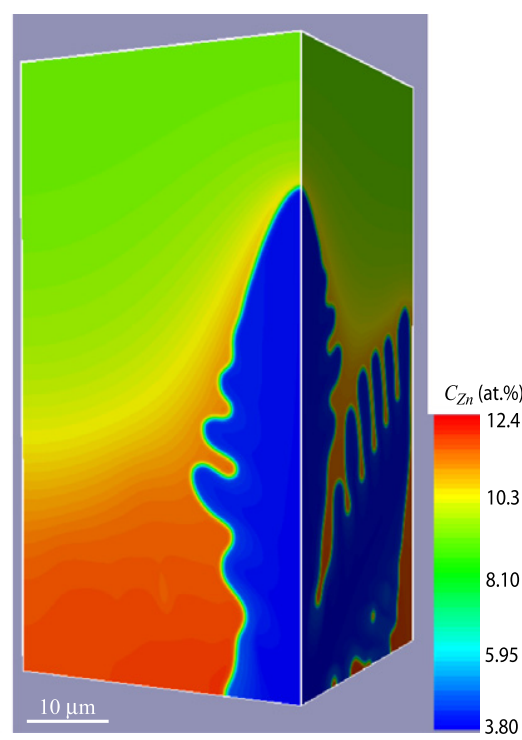

(b)

Fig. 3. 3-D views of the composition profile associated with the growth of a regular $\langle 110\rangle$ dendrite $\left(\theta^{\prime}=90^{\circ}\right)$ in the $(11 \overline{1})$ and $(112)$ planes after $0.11 \mathrm{~s}$ when $\mathbf{j}=0$ (a) and $\mathbf{j} \neq 0$ (b). The domain is $30 \times 60 \times 30 \mu \mathrm{m}^{3}, v_{T}=|\dot{T}| / G=0.5 \mathrm{~mm} \mathrm{~s}^{-1}$.

makes solute to pile up ahead of the groove and slows down its growth until the dendrite tip splits away from the boundary. As $\theta^{\prime}$ increases, this tip splitting occurs at an earlier stage of growth. Once the tip has detached from the boundary, it can grow faster. In between the solid and the twin boundary, a narrow solute-enriched liquid region remains. Due to the 2-D geometry restriction, solute is not able to diffuse along the third axis of the domain. Therefore, it could be argued that the splitting of the dendrite tip away from the boundary is related to this restriction. Such a behavior has already been observed by Niederberger et al. [38] under isothermal conditions for a dendrite growing in thin coatings. The same tendency was reported by the present authors in a recent work, although in that case the anti-trapping term was not included in the phase field formulation [15].
The extension of this situation to three dimensions is shown in Fig. 5, for conditions identical to the 2-D case with $\theta^{\prime}=100^{\circ}$. The simulation domain was $30 \times 100 \times 30 \mu \mathrm{m}^{3}$, but only the bottom half of the entire domain is shown in the two 3-D views of Fig. 5. In Fig. 5a, the blue surface corresponds to the isovalue $\phi=0.5$ after $0.104 \mathrm{~s}$ of growth. The solid attached to the $(11 \overline{1})$ twin plane and to the (112) boundary are identified by red zones $(\phi=1)$, while the liquid is transparent. As the rejected solute can now diffuse in the third dimension, i.e. parallel to the twin plane, the dendrite tip grows faster compared with the 2-D case. This has the following consequences: (i) for the same dihedral angle $\theta^{\prime}$, the solid grows away (splits) from the twin boundary at an earlier stage in three dimensions, i.e. the evolution of the small groove into a doublon-type morphology is faster; and (ii) the width of the liquid channel near the twin boundary, 


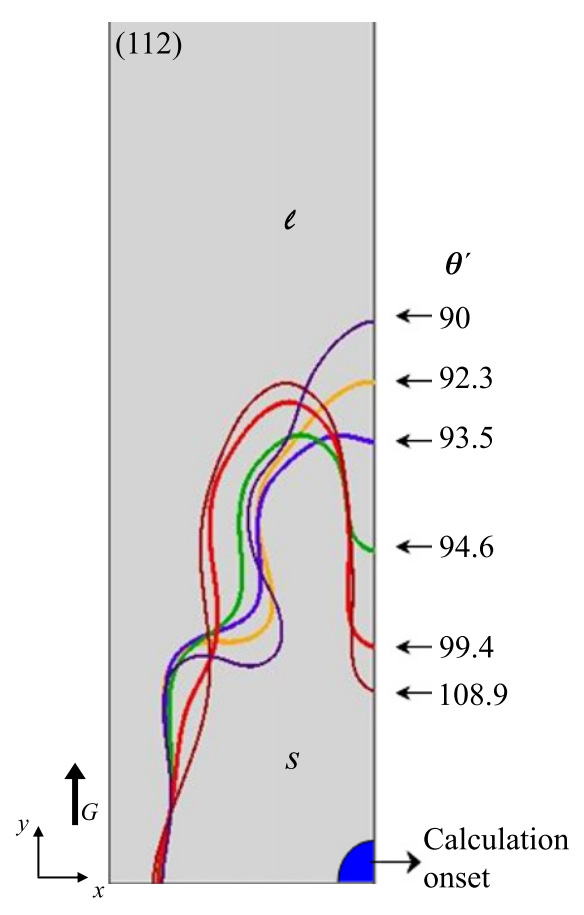

Fig. 4. Effect of the dihedral (wetting) angle on the dendrite tip morphology in two dimensions. The domain is $10 \times 40 \mu \mathrm{m}^{2}$ (only $30 \mu \mathrm{m}$ along the vertical axis are shown), $\quad t=0.11 \mathrm{~s}, v_{T}=|\dot{T}| / G=0.7 \mathrm{~mm} \mathrm{~s}^{-1}, \Delta T=$ $5 \mathrm{~K}, C_{0}=9$ at. $\% \mathrm{Zn}$. The line contours represent the isovalue $\phi=0.5$.

which is of the order of $0.5-2 \mu \mathrm{m}$, depends on the depth of the groove and is reduced by about $25 \%$ as compared with the 2$\mathrm{D}$ case. The dendrite tip instantaneous velocity $v_{\text {tip }}^{d}$, calculated from the length covered by the tip during
$\Delta t=0.008 \mathrm{~s}$, is $0.7 \pm 0.02 \mathrm{~mm} \mathrm{~s}^{-1}$. As it is equal to the imposed speed of the isotherm (see Table 2), this means that the tip has reached a steady state. However, the doublon root is still not growing at the same velocity and thus the overall morphology cannot be considered as steady.

The solute composition field within the (112) and the (11) planes after $0.104 \mathrm{~s}$ of growth is shown in Fig. 5b. The segregation pattern observed around the dendrite side arms in the (112) plane is similar to that of a regular dendrite. However, that in the liquid pool near the twin plane is not: it is due to the solute pile-up already observed in the 2-D simulations. This solute pile-up is also visible all along the twin plane due to the solute rejected by the $\langle 110\rangle$ side arms growing parallel to, but not directly in contact with, the twin plane (see Fig. 5a).

\subsection{Growth kinetics}

The main problem that arises from the simulations presented above is that the dendrite tip reaches the imposed solidification velocity $v_{s} \approx v_{T}$, but the root of the doublon does not. Steady-state growth will be reached when the tip and root of the doublon grow at the same velocity. With no diffusion in the solid and no diffusion parallel to the twin plane, the composition of the liquid at the root of a steady-state doublon should be close to $C_{0} / k_{0}$, i.e. solid composition close to $C_{0}$. In order to reduce the necessary simulation time and domain size to reach a steady state, the solidification interval of the alloy was decreased by decreasing the initial liquid composition $C_{0}$ to 4 at. $\% \mathrm{Zn}$ and increasing the partition coefficient $k_{0}$ to 0.5 , in addition

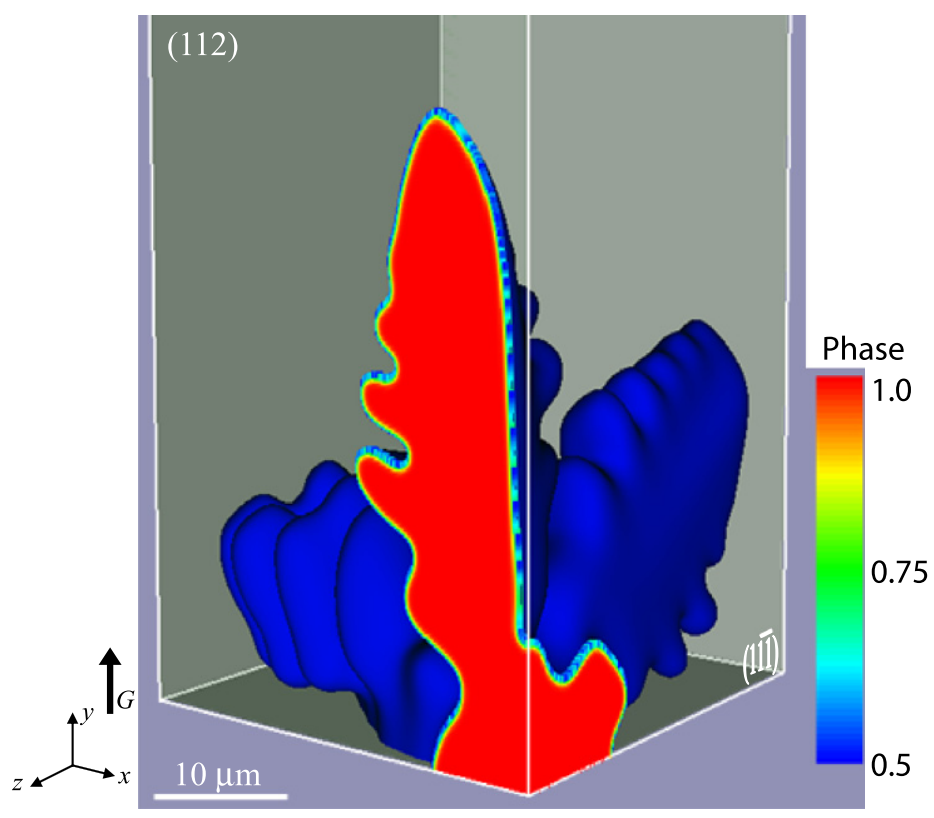

(a)

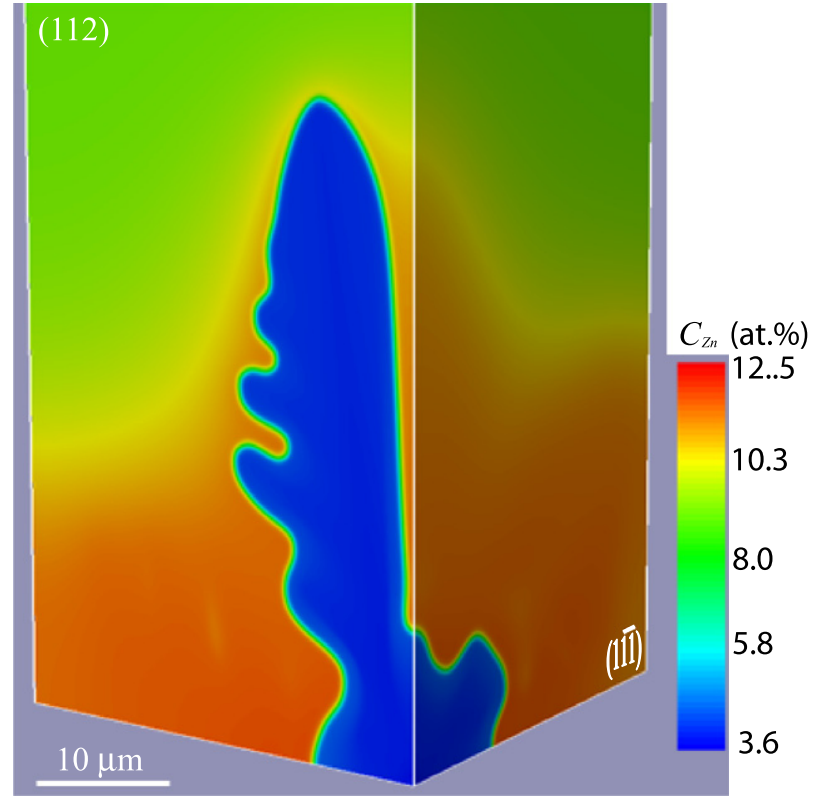

(b)

Fig. 5. (a) 3-D views of the isosurface $\phi=0.5$ after $0.104 \mathrm{~s}$ of simulation for a quarter of a twinned dendrite with $\theta^{\prime}=100^{\circ}$. (b) Associated composition distribution in the (112) and (11 $\overline{1})$ planes. The observed domain is $30 \times 50 \times 30 \mu \mathrm{m}^{3}, t=0.104 \mathrm{~s}, v_{T}=|\dot{T}| / G=0.7 \mathrm{~mm} \mathrm{~s}^{-1}, \Delta T=5 \mathrm{~K}, C_{0}=9$ at. $\% \mathrm{Zn}$. 
to the factor 10 enhancement of the thermal gradient $G$ already made.

Fig. 6 shows two views of the composition fields in the (112) symmetry plane of an $\mathrm{Al}-4$ at.\% $\mathrm{Zn}$ twinned dendrite trunk, taken after $0.152 \mathrm{~s}$ (a) and $0.088 \mathrm{~s}$ (b) of growth. In (a), the doublon is about $106 \mu \mathrm{m}$ in length, i.e. there is a temperature interval of $10.6 \mathrm{~K}$, whereas in (b) it is only $60 \mu \mathrm{m}$ deep (note the change of scale between the two figures). For comparison, the solidification interval of the alloy is $28.6 \mathrm{~K}$. This shows that the doublon is still evolving and has not yet reached a steady shape in (a). The width of the liquid pool between the twinned and untwinned parts of the dendrite is about $3 \mu \mathrm{m}$ near the tip and $0.2 \mu \mathrm{m}$ at the root. Interestingly, below the apparent root of the doublon, small solute-enriched liquid regions are gradually surrounded by the solid phase, first from the side of the (112) plane, then also from the side of the twin plane, as explained in more detail in the next paragraph. The slightly magnified picture taken at $t=0.088 \mathrm{~s}$ (Fig. 6b) corresponds to the time at which a first solute-rich liquid droplet is surrounded by the solid in the (112) plane. This event corresponds to the partial coalescence of the twinned and untwinned regions of the dendrite [39]. As $\gamma_{t}<2 \gamma_{s}$, the coherent boundary is attractive and the coalescence phenomenon is poorly reproduced here for two reasons: (i) imposing a fixed dihedral angle instead of using a multiphase field approach [27] may bias the results; and (ii) coalescence occurs at the scale of the real diffuse solid-liquid interface thickness $\delta$ (a few $\mathrm{nm}$ ), which is nearly three orders of magnitude smaller than the mesh size $\Delta x$ used in the present simulations. Therefore, when the doublon width at the root approaches $\Delta x$ (instead of $\delta$ ), the solid naturally coalesces within this attractive boundary. Nevertheless, even though the mesh size makes coalescence occur earlier, this phenomenon shows that the doublon root is probably never going to be steady due to the unstable nature of the attractive coherent twin boundary.

The mechanism associated with the formation of these small liquid pockets is further illustrated in Fig. 7. Three 3-D projections of the composition distribution near the doublon root in the (112) and (111) planes, at times $t=0.072,0.096$ and $0.112 \mathrm{~s}$, are shown. Solidification of this region is controlled by two mechanisms: (i) solute pile-up in the narrow liquid channel due to the slow lateral

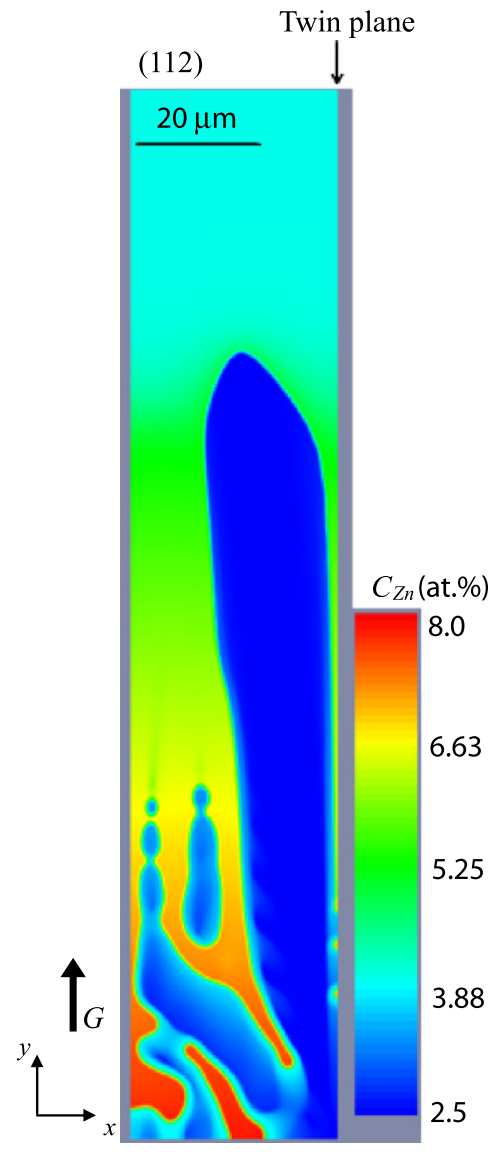

(a)

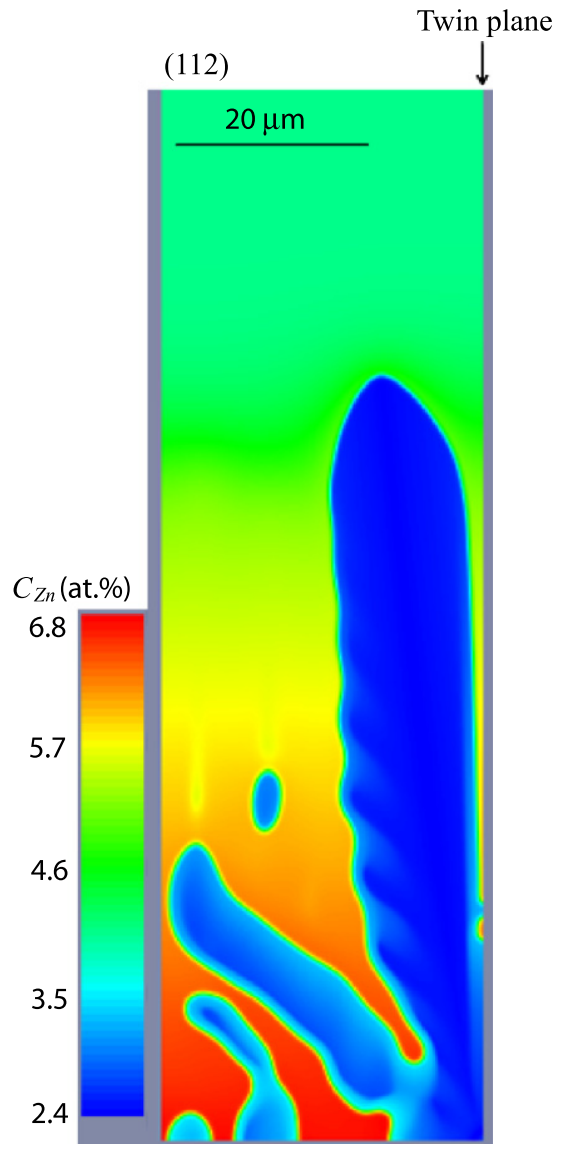

(b)

Fig. 6. (a) Solute distribution map in the (112) plane after $0.152 \mathrm{~s}$ of simulation, as predicted by 3-D simulations; (b) detail of the composition distribution profile after $0.088 \mathrm{~s}$ of simulation. The domain of this simulation is $30 \times 140 \times 34 \mu \mathrm{m}^{3}, v_{T}=|\dot{T}| / G=0.7 \mathrm{~mm} \mathrm{~s}^{-1}, \Delta T=5 \mathrm{~K}, \theta^{\prime}=100^{\circ}$ and $C_{0}=4$ at. $\% \mathrm{Zn}$. 


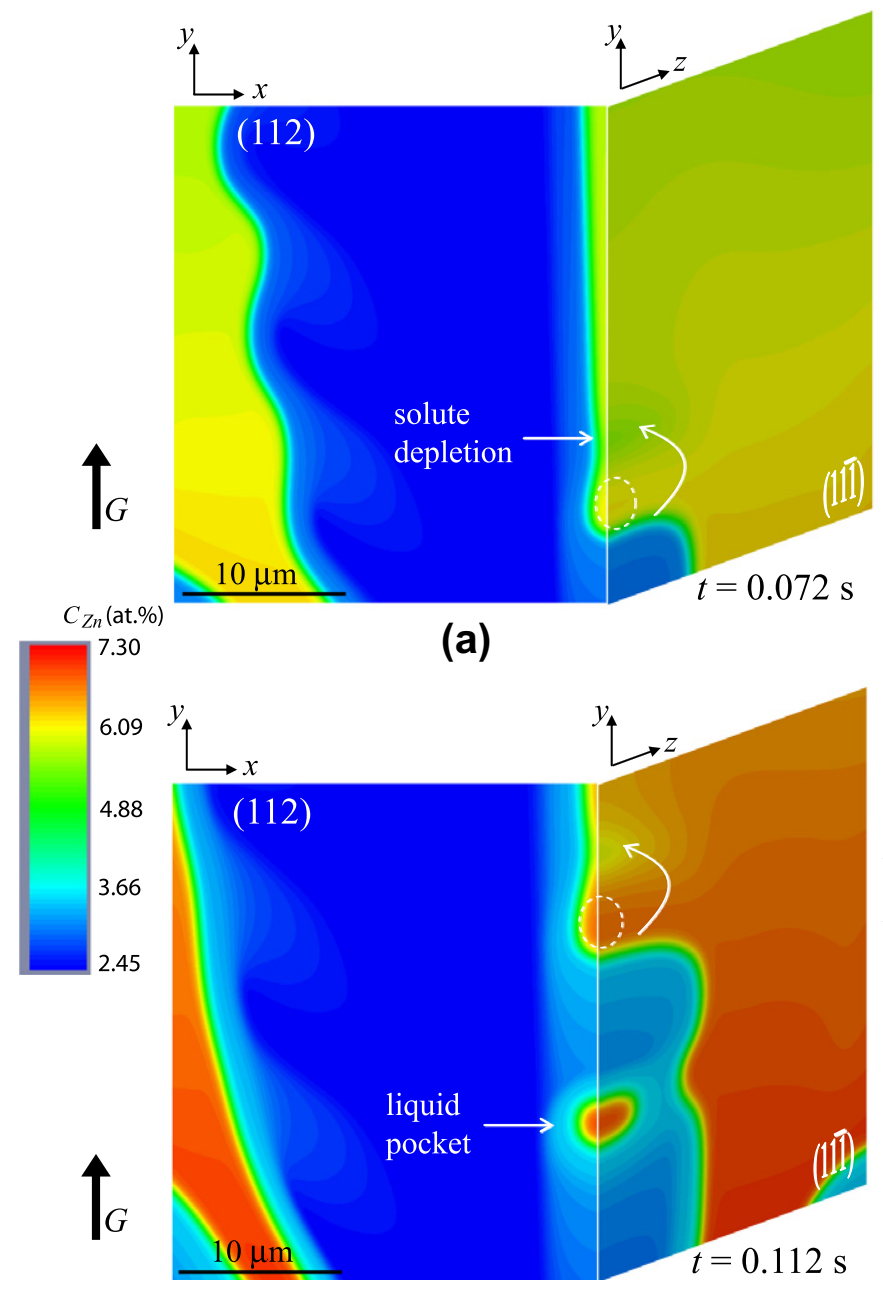

(c)

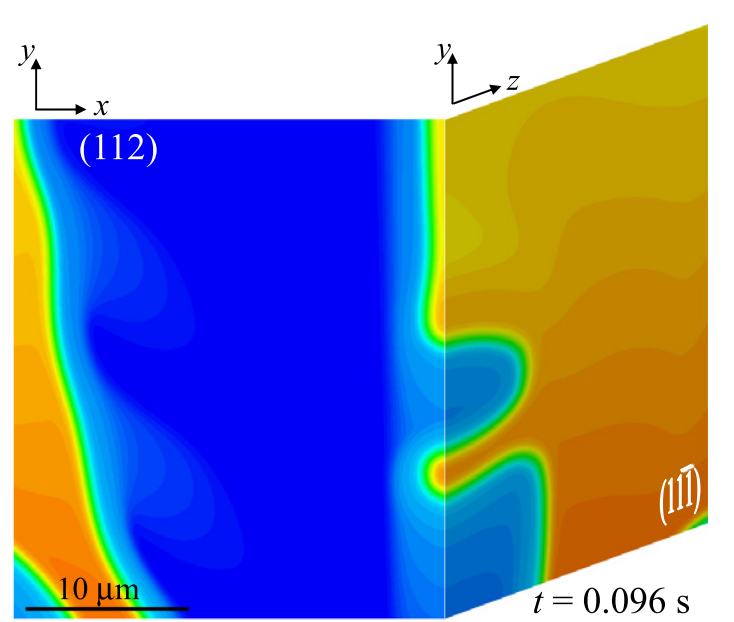

(b)

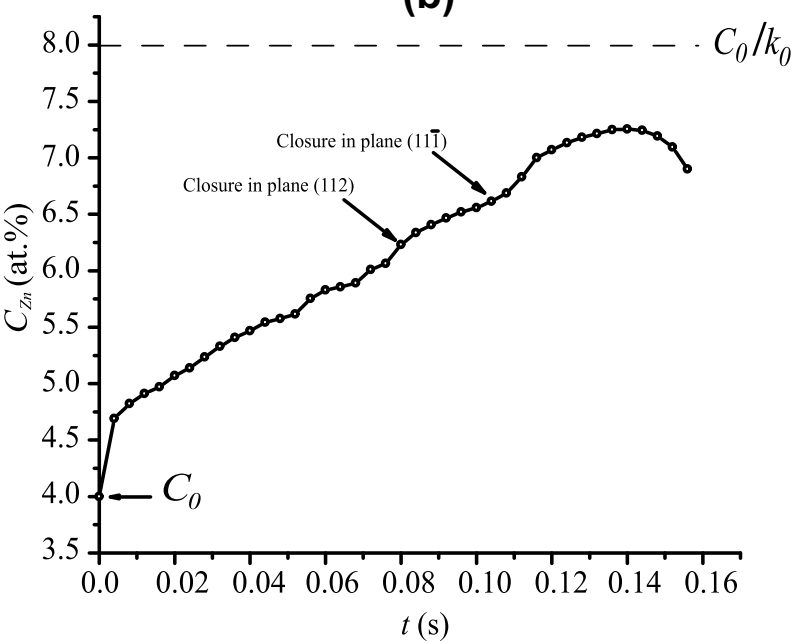

(d)

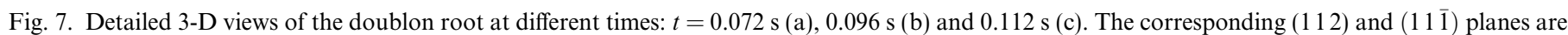
indicated (see text for description); (d) evolution of the liquid composition, $C_{\ell}^{p}$, at a location of the twin plane where a liquid pocket forms.

growth of the two solid parts of the doublon and the growth of the root. Note that the solid-liquid interface at the root of the doublon has a negative curvature in the (112) plane as a result of the imposed equilibrium condition at the triple line; and (ii) solute diffusion in the liquid parallel to the coherent (111) plane and back-diffusion in the solid perpendicular to this plane. These combined effects create a slight solute depletion at about $2 \mu \mathrm{m}$ above the root, as indicated by the small dashed ellipse in Fig. 7a. When the width of this region is comparable to $\Delta x$, the solid coalesces at the boundary (symmetry condition) at the solute depleted region (see Fig. 7b). While this happens, the solid bridge across the twin boundary grows and gradually encapsulates a small liquid pocket at the root of the doublon. This can be observed in Fig. 7c. The diameter of these closed liquid pockets after $0.152 \mathrm{~s}$ of simulation is about $0.5 \mu \mathrm{m}$. A few microns above the liquid pocket, the same mechanism repeats again. The small white arrows in Fig. 7a and $\mathrm{c}$ indicate how the solid periodically encapsulates solute-rich liquid regions at the doublon root. Note the solute gradient in the solid near these liquid pockets, clearly visible in Fig. 7c.

The graph in Fig. $7 \mathrm{~d}$ plots the evolution of the liquid composition at a location of the twin plane where a liquid droplet forms later. It starts at the nominal composition of the alloy, $C_{0}=4$ at. $\% \mathrm{Zn}$, then makes a jump to $4.7 \mathrm{wt} . \%$ when the twinned dendrite tip arrives at this height. Thereafter, it increases steadily as it moves deeper in the liquid pool of the doublon. The first arrow indicates the time at which the solid bridge in the (112) plane forms above this position, while the second arrow corresponds to the complete closure of this liquid region. The composition in this region reaches a maximum at $0.14 \mathrm{~s}\left(C_{\ell}^{p}=7.25 \mathrm{at} . \% \mathrm{Zn}\right)$ and then rapidly decreases as it gradually solidifies due to back diffusion. It should be noted that the maximum value of the liquid pocket composition, $C_{\ell}^{p}$, is below $C_{0} / k_{0}$, the maximum liquid composition given by the linearized equilibrium phase diagram.

The growth velocities of regular and twinned $\langle 110\rangle$ dendrites are compared in Fig. 8 for two alloys. The thermal 


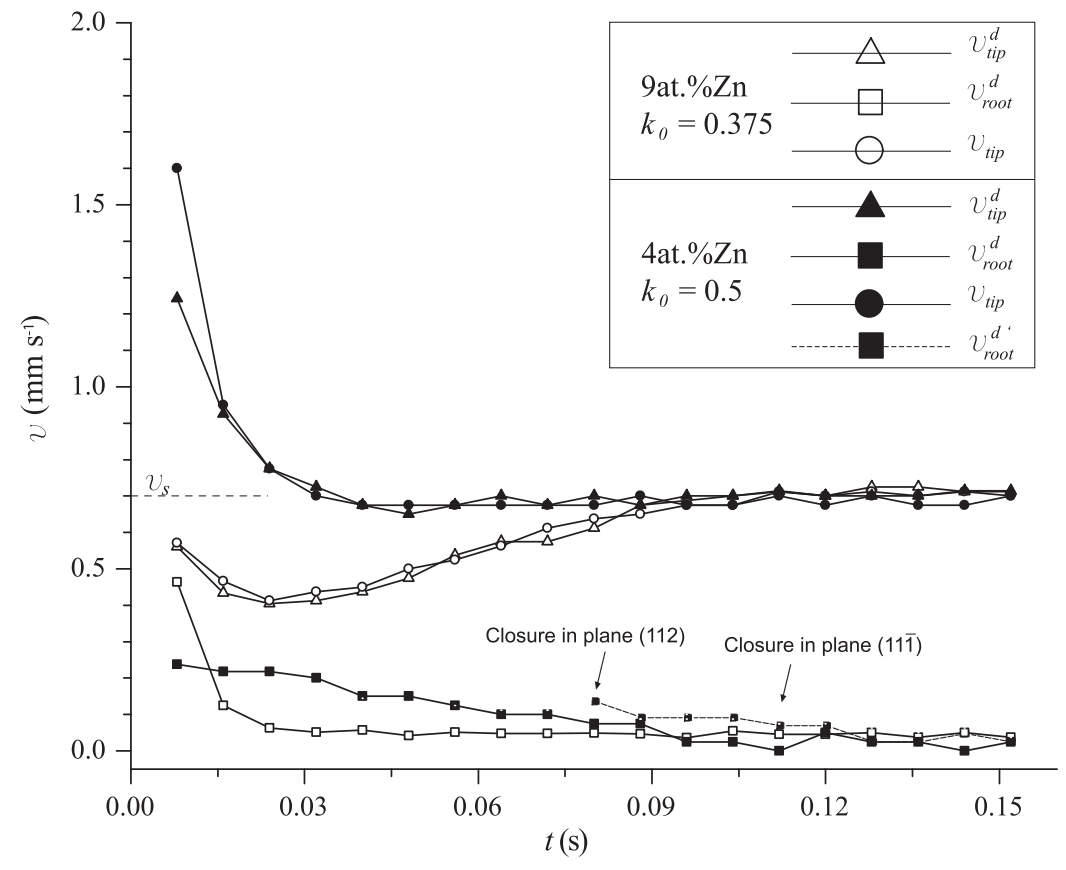

Fig. 8. Comparison of the growth velocities of regular and doublon-like $\langle 110\rangle$ dendrites for two different alloys: Al-9 at. $\% \mathrm{Zn}$ with $k_{0}=0.375$ (open symbols) and $\mathrm{Al}-4$ at. $\% \mathrm{Zn}$ with $k_{0}=0.5$ (filled symbols). Circles: tips of regular dendrites $\left(\theta^{\prime}=90^{\circ}\right)$; triangles: tips of twinned dendrites $\left(\theta^{\prime}=100^{\circ}\right)$; squares: roots of twinned dendrites.

gradient for these simulations is aligned with the vertical axis $(\alpha=0)$. The velocity of regular dendrites $\left(\theta^{\prime}=90^{\circ}\right)$ is shown with circular symbols, whereas that of the tip and root of twinned dendrites $\left(\theta^{\prime}=100^{\circ}\right)$ are displayed with triangular and square symbols, respectively. Empty and filled symbols correspond to an Al-9 at.\% $\mathrm{Zn}$ alloy with $k_{0}=0.375$ and an $\mathrm{Al}-4$ at. $\% \mathrm{Zn}$ alloy with $k_{0}=0.5$, respectively. As can be seen, the time evolution of the tip velocities, $v_{t i p}$ and $v_{t i p}^{d}$, of the regular and doublon-type twinned dendrites are nearly superimposed and reach a steady state $\left(v_{s}=0.7 \mathrm{~mm} \mathrm{~s}^{-1}\right)$ within about $0.1 \mathrm{~s}$. However, the root of the doublon in both alloys never reaches the speed of the isotherm and on the opposite tends toward zero, meaning that the depth of the liquid channel increases continuously. In fact, the doublon root evolves very slowly: while the doublon root of the Al-9 at.\% $\mathrm{Zn}$ alloy reaches a relatively constant growth velocity $\left(v_{\text {root }}^{d} \approx 0.045 \mathrm{~mm} \mathrm{~s}^{-1}\right)$, that of the Al-4 at.\% Zn alloy decreases down to zero when the first

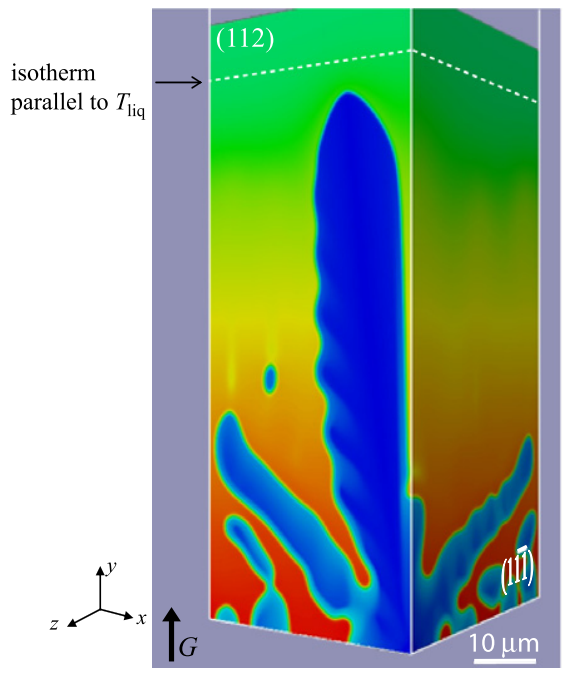

(a)

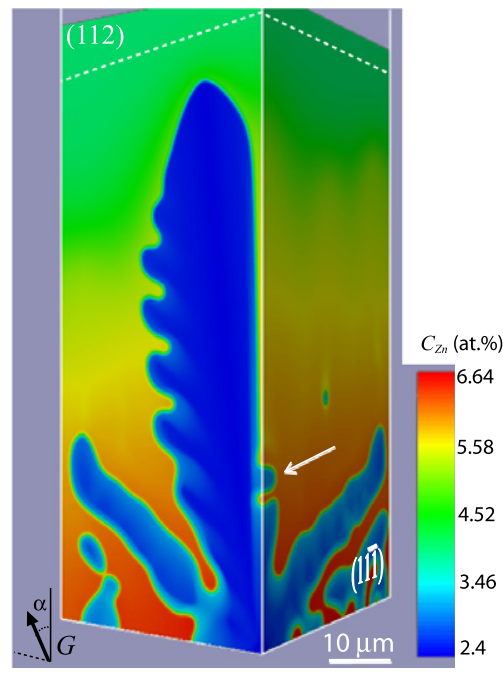

(b)

Fig. 9. Effect of the direction of the thermal gradient $G$. In (a) the $\langle 110\rangle$ dendrite grows parallel to the thermal gradient $\left(\alpha=0^{\circ}\right)$, while in (b) it grows at an angle $\alpha=20^{\circ}$ to $G$. The calculation domain is $30 \times 100 \times 34 \mu \mathrm{m}^{3}$, but only the first $70 \mu \mathrm{m}$ along the $y$ axis is shown, $v_{T}=|\dot{T}| / G=0.7 \mathrm{~mm} \mathrm{~s}{ }^{-1}, \Delta T=5 \mathrm{~K}, \theta^{\prime}=100^{\circ}$, $t=0.072 \mathrm{~s}$. 
liquid pocket forms, then varies between 0 and $0.025 \mathrm{~mm} \mathrm{~s}^{-1}$ simply because the liquid pocket reduces its diameter. The times corresponding to the closure of the liquid pocket, first from the side of the (112) plane, then from the side of the twin plane, have been indicated in the graph. The curve labeled $v_{\text {root }}^{d^{\prime}}$ in Fig. 8 corresponds to the new doublon root formed after coalescence of the twinned and untwinned regions at the twin boundary. The velocity of the new doublon root decreases until a new pocket forms and the actual root "jumps" a few microns ahead along the growth direction.

The corresponding dendrite tip undercoolings for regular and doublon-type twinned dendrites, $\Delta T_{t i p}$ and $\Delta T_{\text {tip }}^{d}$, also evolve towards a steady state. However, the undercooling difference is so small $\left(\left(\Delta T_{\text {tip }}-\Delta T_{\text {tip }}^{d}\right)=+0.03 \mathrm{~K}\right.$ for $\mathrm{Al}-4$ wt. $\% \mathrm{Zn}$ and $-0.03 \mathrm{~K}$ for $\mathrm{Al}-9$ at. $\% \mathrm{Zn}$ ) that no conclusion can be drawn concerning any growth advantage of one morphology over the other based only on diffusion and capillarity considerations. It is known experimentally that other factors, such as convection, are required to favor the formation of twinned dendrites. Besides this factor, the domain size and the time of the simulation should be expanded so that the growth kinetics of the two morphologies can be compared at steady state and for "isolated" tips. Indeed, as the full tip of half of the twinned dendrite grows away from the domain boundary while the half-regular dendrite follows it (see Fig. 4), its solute field interacts more with the opposite boundary of the domain compared with the regular dendrite, and thus its growth kinetics is potentially more affected by the limited size of the calculation domain.

All the simulation results presented so far have assumed that the twinned dendrite trunk grows along a $\langle 110\rangle$ direction perfectly aligned with the thermal gradient $G$. In actual microstructures, twinned dendrites can have a large misorientation with respect to $G$ (as much as $30^{\circ}$ ). Furthermore, the thermal conditions for the calculations shown before, namely $G$ and $\dot{T}$, were set in order to comply with the isotherm velocity $v_{T}$ measured in the early stages of solidification of the DS ingots. However, these parameters decrease drastically within the first few millimeters of the ingot. The effect of the direction of the thermal gradient $G$ was studied for two values of the angle $\alpha$ between the thermal gradient and the vertical axis of the domain (see Fig. 2). In Fig. 9a and $b, 3-D$ views of the composition fields are shown for a quarter of two twinned dendrites growing at an angle $\alpha=0$ and $\alpha=20^{\circ}$ from the thermal gradient, respectively $\left(v_{T}=0.7 \mathrm{~mm} \mathrm{~s}^{-1}, t=0.072 \mathrm{~s}\right)$. Comparing the two pictures, it can be seen that while the liquid channel in the doublon growing parallel to $G$ is still fully open, that of the misoriented twinned dendrite already shows a partially closed liquid pocket (arrow in Fig. 9b). The tip undercooling of the misoriented twinned dendrite, $\Delta T_{\text {tip }}=4.30 \mathrm{~K}$, is also slightly larger than that of the well-aligned one $\left(\Delta T_{t i p}=4.05 \mathrm{~K}\right)$. This can be seen directly in Fig. 9 by looking at their positions with respect to a line parallel to the liquidus isotherm (white dashed line). The undercooling at the root of the doublon in the well-oriented dendrite is $8.38 \mathrm{~K}$ and that of the misoriented dendrite is $8.18 \mathrm{~K}$, while the liquid pocket in Fig. $9 \mathrm{~b}$ (arrow) is $8.52 \mathrm{~K}$ below the liquidus. Thus, it can be concluded that: (i) the tip of a doublon-like $\langle 110\rangle$ twinned dendrite grows at an undercooling that increases with its misorientation, as do regular dendrites, since its velocity is higher (at steady state, $v_{\text {tip }}=v_{T} / \cos \alpha$ ); and (ii) the liquid pockets are formed earlier and the depth of the doublon is reduced when the twinned dendrite is misoriented.

\section{Conclusion}

The stable growth morphology of twinned dendrites has been investigated through 3-D phase-field modeling. The dihedral angle at the triple line between the twinned solid, untwinned solid and liquid has been simulated by an equivalent wetting angle of the dendrite on one of the domain boundaries assumed to be the coherent twin plane. The results of these simulations show that the initial groove associated with the equibrium condition at the triple line rapidly evolves into a doublon morphology in 2-D and 3$\mathrm{D}$ calculations. The liquid channel of the doublon is very narrow (from $3 \mu \mathrm{m}$ near the tip to $0.2 \mu \mathrm{m}$ near the doublon root), and its depth strongly depends on the alloy and on the solidification conditions. At relatively high solidification rates, small liquid droplets form below the doublon root. Their maximum liquid composition remains below $C_{0} / k_{0}$ and the solute-rich liquid in the doublon root is continuously smeared out by liquid diffusion along the twin plane and by back-diffusion. The composition gradient in the solid near the twin plane is also smeared out by backdiffusion. The formation of these liquid pockets occurs earlier when the dendrite growth direction is misoriented with respect to the thermal gradient.

Although done in three dimensions, these simulations have nevertheless some limitations: the tip is not totally free due to the size of the domain, the doublon itself is not at steady state even if the tip is, the formation of the liquid pockets at the root of the doublon by coalescence is enhanced by the too large mesh size, convection, which is a pre-requisite for twinned dendrite formation, is not accounted for, etc. Nevertheless, these findings strongly support the doublon conjecture first made by Henry [8] for the morphology of a twinned dendrite tip. Experimental evidence supporting the doublon conjecture is presented in the following companion paper [10].

\section{Acknowledgements}

The authors would like to thank the staff of the MIZAR computer facility of the Ecole Polytechnique Fédérale de Lausanne (EPFL). The collaboration of Dr. Aurèle Mariaux for building the visualization tools of the simulation results is also gratefully acknowledged. 


\section{References}

[1] Salgado-Ordorica MA, Burdet P, Cantoni M, Rappaz M. Acta Mater 2011. doi:10.1016/j.actamat.2011.04.034.

[2] Henry S, Jarry P, Jouneau P-H, Rappaz M. Metall Mater Trans A 1997;28A:207-13.

[3] Henry S, Jarry P, Rappaz M. Metall Mater Trans A 1998;29A:2807-17.

[4] Henry S, Minghetti T, Rappaz M. Acta Mater 1998;46(18):2495-501.

[5] Herenguel J. Rev Metall 1948;45(5):339-46.

[6] Morris RL, Carruthers JR, Plumtree A, Winegard WC. AIME Metall Soc Trans 1966;236(9):1286-91.

[7] Spear RE, Craig RT, Howle CR. J Metals 1971;23:42.

[8] Henry S. PhD thesis, Ecole Polytechnique Fédérale de Lausanne; 1999.

[9] Salgado-Ordorica MA, Rappaz M. Acta Mater 2008;56:5708-18.

[10] Salgado-Ordorica MA, Phillion A, Rappaz M. Metall Mater Trans; submitted for publication.

[11] Bolling GF, Tiller WA. J Appl Phys 1960;31(8):1345-50.

[12] Jones DRH, Chadwick GA. J Cryst Growth 1971;260:291-300.

[13] Chalmers B. Principles of solidification. New York: John Wiley \& Sons; 1964.

[14] Eady JA, Hogan LM. J Cryst Growth 1974;23:129-36.

[15] Salgado-Ordorica MA, Desbiolles J-L, Rappaz M. In: Crockcroft S, Maijer D, editors. Modelling of casting welding and advanced solidification processes, vol. XII. TMS; 2008. p. 545-52.

[16] Wood HJ, Hunt JD, Evans PV. Acta Mater 1997;45(2):569-74.

[17] Napolitano RE, Liu S. Phys Rev B 2004;70:214103.

[18] Akamatsu S, Faivre G, Ihle T. Phylos Rev E 1995;51(5):4751-72.

[19] Singer HM, Singer I, Bilgram JH. JOM Dendrite Growth Process 2007;59(7):77-80.
[20] Brenner E, Muller-Krumbhaar H, Saito Y, Temkin DE. Phys Rev E 1993;47:1151-5.

[21] Ihle T, Müller-Krumbhaar H. Phys Rev E 1994;49(4):2972-91.

[22] Amar MB, Brener E. Phys Rev Lett 1995;75:561-4.

[23] Abel T, Brener EA, Mueller-Krumbhaar H. Phys Rev E 1997;55:7789-92.

[24] Warren JA, Boettinger WJ. Acta Metall Mater 1995;43(2):689-703.

[25] Karma A, Rappel W-J. Phys Rev E 1996;53(4).

[26] Boettinger WJ, Warren JA, Beckermann C, Karma A. Annu Rev Mater Res 2002;32:163-94.

[27] Tiaden J, Nestler B, Diepers HJ, Steinbach I. Physica D 1998;115:73-86.

[28] Kobayashi R, Warren JA, Craig Carter W. Physica D 2000;140:141-50.

[29] Niederberger C, Michler J, Jacot A. Phys Rev E 2006;74:021604.

[30] Fehlner WR, Vosko SH. Can J Phys 1976;54:2159-69.

[31] Friedli J, Mariaux A, Gonzales F, Rappaz M. In: Crockcroft S, Maijer D, editors. Modelling of casting welding and advanced solidification processes, vol. XII. TMS; 2009. p. 627-34.

[32] Echebarria B, Folch R, Karma A, Plapp M. Phys Rev Lett 2004;70:061604.

[33] Haxhimali T, Karma A, Gonzales F, Rappaz M. Nat Mater 2006;5.

[34] Karma A. Phys Rev Lett 2001;87(11):115701.

[35] Sémoroz A, Henry S, Rappaz M. Metall Mater Trans A 2000;31A:487-95.

[36] Mariaux A. PhD thesis, EPFL No. 4646; 2010.

[37] Mariaux A. Internal report LSMX, EPFL; 2007.

[38] Niederberger C. PhD thesis, EPFL; 2007.

[39] Rappaz M, Jacot A, Boettinger WJ. Metall Mater Trans A 2003;34A:467-79. 